\title{
A low-cost, endoscopic, digital, still and video photography system for ENT clinics
}

\author{
B W Howes, C Repanos*
}

\begin{abstract}
Image capture systems that display and record endoscopic images are important for documentation and teaching. We have modified a universal serial bus microscope to couple with most clinical endoscopes used in our practice. This very economical device produces images suitable for teaching, and potentially for clinical use. The implications of this could be significant for teaching, patient education, documentation and the developing world.
\end{abstract}

Key words: Camera; Imaging; Endoscope; Teaching

\section{Introduction}

Image capture systems that display and record endoscopic images are important for documentation and teaching. ${ }^{1}$ Many ENT clinics have only one purpose-built system, and the majority of clinic rooms have no image recording facilities.

Home-made methods for attaching consumer digital still cameras to endoscopes have been described. ${ }^{2-5}$ Most record only still images, do not provide a 'live' image preview and are expensive, and some are large.

We have modified a cheap consumer electronic microscope to couple with endoscopes that have a standard eyepiece. Video and still images can be displayed and recorded on any universal serial bus (USB) equipped computer (Figure 1) and (Figure 2).

\section{Method}

Webcams are cheap, miniature, digital video cameras connected to a computer, which capture images of the user. The Veho ${ }^{\mathrm{TM}}$ VMS-001USB microscope (Veho HQ, Hampshire, UK; www.veho-uk.com) has a similar function to a webcam, but is designed for high magnification.

We used part of a disposable camera-head drape (Fairmont Medical(C) DCC 8007 disposable camera drape (Bayswater, Victoria, Australia)) as a cheap method of attaching the USB microscope to an endoscope. The fitting is suitable for clinical endoscopes equipped with the common standard $32 \mathrm{~mm}$ eyepiece.

The USB microscope and endoscope adapter required modification. More details are available online at http:// www.usbendoscopy.co.uk.

The camera software runs on most operating systems, including the latest 'netbook' low cost compact laptop computers. When combined with a battery-powered endoscopy light source, the system is exceptionally portable. (a)

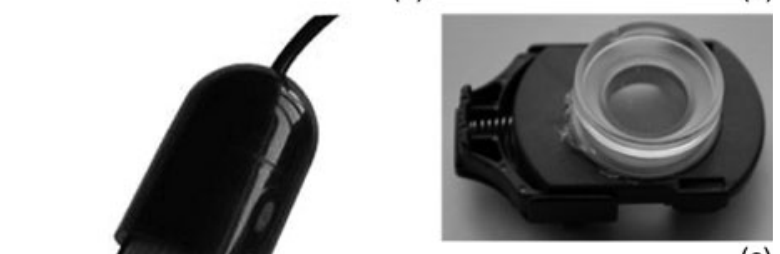

(c)

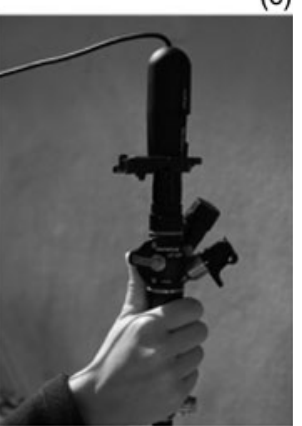

FIG. 1

(a) Veho ${ }^{\mathrm{TM}}$ VMS 001 USB Microscope. (b) Fairmont Medical 8007 disposable camera drape (polythene removed). (c) Universal serial bus camera head.

We asked 30 colleagues to compare video images (of nasendoscopy performed on one of the authors) recorded on the USB microscope with images from a professional image capture system. They reported that the USB system produced images suitable for teaching and clinical work. We do not advise its use as a diagnostic imaging tool, as we are awaiting clearance for clinical purposes from the Medicines and Healthcare Products Regulatory Agency; however, the device has been cleared as safe by

From the Departments of Anaesthesia and *Otolaryngology, Royal United Bath Hospital, UK.

Presented at the Group of Anaesthetists in Training meeting, 4 July 2009, Cambridge, UK.

Awarded the Association of Anaesthetists of Great Britain and Ireland Registrar's Prize (President's Medal).

Accepted for publication: 17 September 2009. First published online 22 December 2009. 


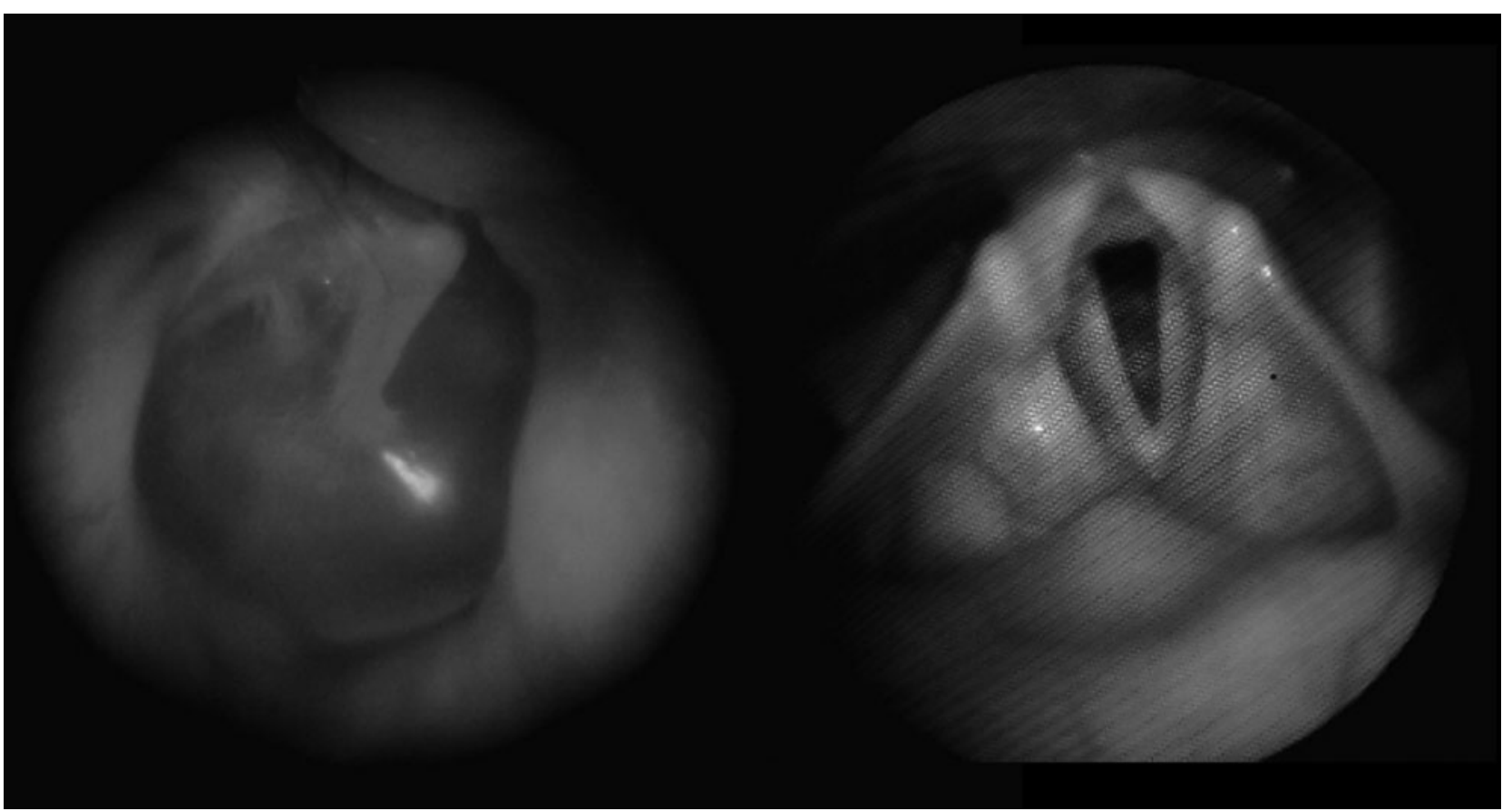

FIG. 2

Example of still images obtained via universal serial bus microscope.

our hospital's medical equipment department. A video is available online at http://www.vimeo.com/6006462.

\section{Conclusions}

The low cost USB endoscopy camera described has excellent potential for teaching, patient education, documentation and use in the developing world. The image obtained may be useful for demonstrating pathology in the out-patient clinic and in-patient ENT consultation, and for aiding fibre-optic intubation during anaesthesia.

\section{References}

1 Tabaee A, Johnson PE, Anand VK. Office based digital video endoscopy in otolaryngology. Laryngoscope 2006; 116: $1523-5$

2 Judd O, Repanos C. Digital photography in otolaryngology. The Otolaryngologist 2007;1:127-31

3 Barr GD. Low cost digital endoscopic photography. $J$ Laryngol Otol 2009;123:453-6
4 Melder PC. Simplifying video endoscopy: inexpensive analog and digital video endoscopy in otolaryngology. Otolaryngol Head Neck Surg 2005;132:804-5

5 Pothier DD, Hajiioannou J, Grant DG, Repanos C, Kalk N. A simple and inexpensive technique of recording operative microscopic and endoscopic video directly to your computer. Clin Otolaryngol 2007;32:498-500

Address for correspondence:

Mr C Repanos,

Specialist Registrar in Otolaryngology,

Royal United Bath Hospital,

Combe Park,

Bath BA1 3NG, UK.

E-mail: costa@repanos.com

Mr C Repanos takes responsibility for the integrity of the content of the paper.

Competing interests: None declared 